\title{
miR-200c-141 Enhances Sheep Kidney Cell Reprogramming into Pluripotent Cells by Targeting ZEB1
}

\author{
Yunfeng Zhang ${ }^{1,2,3, *}$, Yanhua $\mathrm{He}^{3, *}$, Peng $\mathrm{Wu}^{4, *}$, Shengwei $\mathrm{Hu}^{4}$, Yanyan Zhang ${ }^{3}$, Chuangfu Chen ${ }^{1,2}$ \\ ${ }^{1}$ College of Animal Science and Technology, Shihezi University, Xinjiang, China \\ ${ }^{2}$ Collaborative Innovation Center for Prevention and Control of High Incidence Zoonotic Infectious, Shihezi, China \\ ${ }^{3}$ State Key Laboratory of Sheep Genetic Improvement and Healthy Production/Xinjiang Academy of Agricultural and Reclamation \\ Sciences, Xinjiang, China \\ ${ }^{4}$ College of Life Technology, Shihezi University, Xinjiang, China
}

Background and Objectives: Sheep-induced pluripotent stem cells (siPSCs) have low reprogramming efficiency, thereby hampering their use in biotechnology and agriculture. Several studies have shown that some microRNAs play an important role in promoting somatic reprogramming in mouse and human. In this study, we investigated the effect of miR-200c-141 on somatic reprogramming in sheep and explored the mechanism of promoting the reprogramming. Methods and Results: The lentivirus system driven by tetracycline (TET)-on carrying Oct4, Sox2, c-Myc, Klf4, Nanog, Lin28, hTERT, and SV40LT (OSKMNLST) could reprogram sheep kidney cells into pluripotent cells. Overexpression of miR-200c-141 in combination with OSKMNLST could significantly improve the efficiency of sheep iPSC generation $(\mathrm{p}<0.01)$. Sheep iPSCs derived from miR-200c-141 showed embryonic stem cell (ESC)-like pluripotent properties, were positive for alkaline phosphatase and some pluripotent markers by quantitative real-time PCR (qRT-PCR) and immunofluorescence, and were able to differentiate into three germ layers in vitro. Oar-miR-200c was transfected into HEK293FT cells and was able to target the zinc finger E-box-binding homeobox 1 (ZEB1) 3'UTR using dual luciferase reporting analysis. Overexpression of oar-miR-200c in SKCs significantly reduced the expression of ZEB1, but increased the expression of E-cadherin by qRT-PCR and western blotting analysis.

Conclusions: These results suggest that miR-200c-141 can promote the reprogramming of sheep somatic cells to iPSCs, and oar-miR-200c targeted ZEB1 3'UTR, significantly decreased expression of ZEB1, and increased expression of E-cadherin. Oar-miR-200c may improve the MET process by affecting the TGF- $\beta$ signaling pathway, thus improving the efficiency of somatic cell reprogramming in sheep.

Keywords: Sheep, iPSC, miR-200c, ZEB1, E-cadherin

Received: April 22, 2021, Revised: May 31, 2021,

Accepted: June 11, 2021, Published online: August 31, 2021

Correspondence to Chuangfu Chen

College of Animal Science and Technology, Shihezi University,

No. 221 North 4 Road, Shihezi, Xinjiang 83200, China

Tel: +86-993-2057805, Fax: +86-993-2058839

E-mail: chuangfu_chen@163.com

${ }^{*}$ These authors contributed equally to this work.

(a) This is an open-access article distributed under the terms of the Creative Commons Attribution Non-Commercial License (http://creativecommons.org/ licenses/by-nc/4.0/), which permits unrestricted non-commercial use, distribution, and reproduction in any medium, provided the original work is properly cited.

Copyright (c) 2021 by the Korean Society for Stem Cell Research

\section{Introduction}

Induced pluripotent stem cells (iPSCs) are an emerging stem cell manufacturing technology. The classic approach is to transfer a specific combination of transcription factors into somatic cells and reprogram these into undifferentiated pluripotent cells. Similar to embryonic stem cells (ESCs), iPSCs have the ability of self-renewal and pluripotency, and can differentiate into three germ layers under appropriate induction conditions $(1,2)$. Cell reprogramming is a key factor in the transformation of so- 
matic cells into stem cells.

Sheep is a very important domestic animal. The use of iPSCs for precise genetic engineering operations to produce genetically modified sheep can improve their production traits and disease resistance and provide a new way for sheep breeding improvement. The earliest sheepinduced pluripotent stem cell (siPSC) generation involved the introduction of the exogenous OSKM (Oct4, Sox2, c-Myc, Klf4) (3) or OSKMNLST (Oct4, Sox2, c-Myc, Klf4, Nanog, Lin28, SV40LT and hTERT) (4) factors into sheep fetal fibroblasts to produce pluripotent stem cell (PSC)like cells in primed or naïve states using Dox-induced lentiviral vector system. However, stemness maintenance of these siPSCs relied on continuous exogenous transgene expression, and removal of Dox in the culture medium triggers cell differentiation $(3,4)$. Only two studies on naïve PSC-like siPSCs reported that all transgenes were silenced in siPSCs $(5,6)$. In addition, the use of retroviral vectors to transfect OSKM can reprogram sheep embryonic fibroblasts into siPSCs $(5,7)$. These siPSCs could form chimeric inner cell masses (ICMs) after being injected into early sheep blastocysts (5), but the contribution rate of siPSCs to chimeric offspring is very low, integrating into host tissues less than $0.1 \%$ (7). The induction efficiency of siPSCs was between $0.002 \%$ and $0.5 \%(4,5)$. Our laboratory earlier determined that knockdown of p53 and overexpression of ASF1 could increase the reprogramming efficiency to $1 \%$ (6). In short, siPSCs have the problems of low induction efficiency and difficulty in silencing exogenous genes. It was possible that these siPSCs had not been completely reprogrammed (8). Therefore, it is essential to establish an efficient and safe reprogramming method to obtain naive siPSCs with continuous self-renewal ability.

MicroRNAs (miRNAs) are small non-coding RNAs with a length of 21 25 nucleotides (nt) that can bind to complementary mRNA sequences and inhibit their expression through mRNA cleavage or protein translation (9). miRNAs can play an important regulatory role in the gene transcription network that guides cell fate decisions. Studies have shown that miRNAs and transcription factors are involved in the maintenance of cell functions by regulating specific gene expression patterns in stem cell self-renewal, lineage-specific differentiation and somatic cell reprogramming (10). Specific miRNAs are highly expressed in ESCs and play a key role in controlling pluripotencyrelated genes (11-13). The miRNA-mediated reprogramming technology has shown that PSC induced by miRNAs has higher reprogramming efficiency and lower tumorigenicity (14). Even in the absence of other transcription factors, only the introduction of mature miRNA could re- program somatic cells into siPSCs (15). Studies have shown that the miR-200 family is directly activated by Oct4 and Sox 2 in mouse, which could help fibroblasts overcome the mesenchymal-epithelial transformation (MET) barrier and promote the production of iPSCs (16). miR-302s, miR-369s and miR-200c could promote the reprogramming of somatic cells in mouse and human (17). Currently, a dozen of miRNAs specifically expressed in ESCs of mouse and human have been discovered. These miRNAs mainly come from four families: miR-290, miR-302, miR-372, and miR-200 (18). Therefore, combining miRNAs with traditional induction methods may establish an efficient system for iPSCs generation.

In the study, we aimed to investigate whether miR-200c also had a promoting effect on sheep iPSC generation and to identify the pluripotency of the obtained sheep iPSCs. The mechanism of miR-200c in the reprogramming process of sheep somatic cells was preliminarily explored. This study not only established a rapid and highly efficient iPSC reprogramming system but also provided a platform to explore the culture conditions for ovine iPSCs and ESCs.

\section{Materials and Methods}

\section{Cell culture}

Sheep kidney cells (SKCs) were isolated from Chinese Merino fetuses ( $\sim 45 \mathrm{~d})$ that were derived from a livestock slaughterhouse in Shihezi City (China) and cultured in Dulbecco's modified Eagle medium (DMEM; Gibco) supplemented with $10 \%$ fetal bovine serum (FBS; Gibco), 1\% non-essential amino acids (NEAA; Gibco), penicillinstreptomycin $(100 \times$, Coolaber, China), and $100 \mu \mathrm{g} / \mathrm{ml}$ Primocin (Invitrogen). ICR mouse embryonic fibroblasts (MEFs; Cyagen, China) were treated with mitomycin C (Sigma) as feeder layers and cultured in SKC medium without Primocin. Sheep iPSCs were cultured on inactivated ICR MEFs in DMEM/F12 medium (Gibco) supplemented with 20\% KnockOut Serum Replacement (Gibco), 1\% NEAA, 1\% GlutaMAX (Gibco), $0.1 \mathrm{mM} \beta$ mercaptoethanol (Sigma), $4 \mathrm{ng} / \mathrm{ml}$ basic fibroblast growth factor (bFGF; Peprotech), $1 \mu \mathrm{g} / \mathrm{ml}$ doxycycline hyclate (DOX; Sigma), $50 \mu \mathrm{g} / \mathrm{ml}$ vitamin C (Vc; Sigma), and 1 $\mathrm{mM}$ valproic acid (VPA; Sigma). Embryoid body (EB) formation medium (Cyagen, China) was composed of $440 \mathrm{ml}$ basal medium, $50 \mathrm{ml}$ fetal bovine serum, $5 \mathrm{ml} \mathrm{NEAA}, 500$ $\mu 1$ 2-mercaptoethanol, $5 \mathrm{ml} \mathrm{L}$-glutamine, and $5 \mathrm{ml}$ penicillin-streptomycin. 


\section{Lentivirus generation}

Eight lentiviral vectors containing human defined transcription factors (lv-efla-egfp-tre-Oct4/Sox2/Klf4/c-Myc/ Nanog/Lin28/hTERT/SV40LT), Tet-on vector lv-EF1artTA-IRES-GFP, and two helper plasmids (pVSVG and $\Delta$ 8.91) were kindly provided by Prof. Lei Xiao (4). The lentiviral vector plenti $4.1 \mathrm{Ex}$ miR200c-141 (\#35534) and helper plasmids pSPAX2 (\#12260) and pMD2.G (\#12259) were provided by addgene. Ten lentiviruses were packaged in HEK293FT cells (Cell Bank, Chinese Academy of Sciences) using the calcium phosphate method according to the high efficiency transfection kit instructions (Biowit, China). The dosage of plasmids was $12 \mu \mathrm{g}$ of the transcription factor/or Tet-on vector, plus $4 \mu \mathrm{g}$ pVSVG and $8 \mu \mathrm{g} \Delta 8.91$ per $10-\mathrm{cm}$ culture plate, and $12 \mu \mathrm{g}$ plenti 4.1 Ex miR200c-141, $9 \mu \mathrm{g} \mathrm{pSPAX2,} \mathrm{and} 3.5 \mu \mathrm{g}$ pMD2.G. All lentiviruses were concentrated with 100 NMWL Amicon Ultra-15 (Millipore, USA; 6,000 g, $30 \mathrm{~min}$ ) and titrated with a series of dilutions by infecting HET293FT cells.

\section{Generation of siPSCs}

We plated 50,000 SKCs (P3) per well into a six-well plate and infected these with lentivirus at 10 multiplicity of infections $(\mathrm{MOI}=10)$ in the presence of polybrene (final concentration $8 \mu \mathrm{g} / \mathrm{ml}$ ) (6). The reprogramming process was divided into two groups: group A with 8 factors (Oct4/Sox2/Klf4/c-Myc/Nanog/Lin28/hTERT/SV40LT) and group B with 8 factors + miR200c-141. Fresh SKC medium was replaced $24 \mathrm{~h}$ post-infection. The infection effect was evaluated under inverted fluorescence microscope $48 \mathrm{~h}$ post-infection. SKCs were digested with TryPLE (Gibco, USA) and inoculated into 6-well plates coated with MEF feeder layer cells for further culture after 48 h post-infection. Reprogrammed cells were digested by TrypLE and were passaged in a 6-well plate with feeder layer cells in a ratio of $1: 8$ for further culture on D10 post-infection. On D16 post-infection, the typical colonies with clear boundaries and protrusions were picked out with glass needles, sub-cultured into 96-well plates, and then expanded in culture. The remaining positive colonies were counted using alkaline phosphatase (AP) staining. Reprogramming efficiency $=$ (Number of alkaline phosphatase (AP)-positive colonies/Number of initially infected cells) $\times 100 \%$.

\section{AP staining}

An AP kit (Beyotime, China) was used for preliminary identification of siPSCs by AP staining. The steps were as follows: the cells were fixed with $4 \%$ paraformaldehyde
(PFA) for 10 min, followed by the addition of BCIP/NBT staining solution (400 $\mu 1$ per well). The cells were stained at room temperature for $1 \mathrm{~h}$ or longer in the dark, and staining intensity was assessed under an inverted microscope.

\section{Immunofluorescence}

The siPSCs were identified after typical colony formation. The differentiation ability of EB was measured on the 7th day in a 24-well cell culture plate. Cells were fixed with $4 \%$ paraformaldehyde for $20 \mathrm{~min}$, permeabilized with $0.2 \%$ Triton X-100 for $10 \mathrm{~min}$, and then blocked with $2 \%$ BSA for $30 \mathrm{~min}$ at room temperature. The primary antibodies against Sox2 (1:200, Abcam), Oct-3/4 (1:100, Santa Cruz), Nanog (1:100, Santa Cruz), Rex-1 (1:100, Santa Cruz), SSEA-1 (1:20, Developmental Studies Hybridoma Bank), E-cadherin (1:100, BD), Tra-1-60 (1:200, Millipore), Tra-1-81 (1:200, Millipore), smooth muscle actin (SMA, 1:200, Santa Cruz), $\beta$-tubulin (Tuj-1, $1: 100$, Santa Cruz), and human Sox17 (Sox17, $1: 100, \mathrm{R} \& \mathrm{D})$ were added and incubated overnight at $4^{\circ} \mathrm{C}$. Cy3-labeled anti-mouse IgG $(1: 500$, Beyotime, China) and anti-mouse $\operatorname{IgM}(1: 500$, Bioss, China) secondary antibodies were added for $1 \mathrm{~h}$ at $37^{\circ} \mathrm{C}$ for detection of target proteins. Nuclei were stained with DAPI (1 : 1,000, Sigma) for $5 \mathrm{~min}$.

\section{Real-time PCR}

The cells were lysed with TRlzol reagent (Life, USA). Total RNA was extracted using an Ultrapure RNA Kit (Cwbio, China), and RNA concentration was determined using a Nano-Drop 200c system (Thermo, USA). RNA was reverse-transcribed into a cDNA using PrimeScript ${ }^{\mathrm{TM}} \mathrm{RT}$ reagent kit (Takara, China). Finally, the cDNA was amplified using a $2 \times$ SYBR Green I Master Kit (Roche, Switzerland) on a Roche LightCycler 480 instrument. Relative mRNA expression levels were analyzed by the $2^{-\Delta \Delta \mathrm{Ct}}$ method; $-\Delta \Delta \mathrm{Ct}$ (Treatment $-\mathrm{Control})=\left(\mathrm{Ct}_{\text {Target }}\right.$ gene $\left.-\mathrm{Ct}_{\text {Gapdh }}\right)$ Treatment $-\left(\mathrm{Ct}_{\text {Target gene }}-\mathrm{Ct}_{\text {Gapdh }}\right)$ Control $(19)$. The PCR primers and profile used to analyze the pluripotent gene of siPSCs were as previously reported (4). Primary SKCs were used as negative control, and GAPDH was employed as internal reference gene. The relative expression levels of zinc finger E-box-binding homeobox 1 (ZEB1) and E-cadherin mRNA were also determined by quantitative real-time PCR (qRT-PCR). The primer sequences were as follows: ZEB1-F 5'-ACTGCAGTCATCTACCCCG A-3', ZEB1-R 5'-CGCTCAAGC TGGGTTCTGTA-3'; E-Cadherin-F 5'-T GGAACTCAGACGGAAGTGC-3', E-Cadherin-R 5'-TCT GGACCAGCGACTTAGGA-3'; and GAPDH-F 5'-ACGGG 
AAGCTCACTGGCATGG-3', GAPDH-R 5'-GCCAGCCC CAGCATCGAAG-3'. Briefly, SKC cells were passaged into 12 -well plates. When cell confluency was about $70 \%$, oar-miR-200c mimics/or inhibitor/or NC $(20 \mathrm{nM})$ at 2.5 $\mu 1 /$ well was transfected into cells using Lipofectamine 2000 (Thermo, USA). At $48 \mathrm{~h}$ after transfection, RNA was extracted for analysis. The PCR reaction was performed at $95^{\circ} \mathrm{C}$ for $5 \mathrm{~min}$; followed by 40 cycles of $95^{\circ} \mathrm{C}$ for $30 \mathrm{~s}$, $62^{\circ} \mathrm{C}$ for $30 \mathrm{~s}$, and $72^{\circ} \mathrm{C}$ for $30 \mathrm{~s}$; and a final elongation at $72^{\circ} \mathrm{C}$ for $5 \mathrm{~min}$. SKCs without miRNA transfection were used as negative control. miRNA mimics/inhibitors/NC was synthesized (GenePharma, China). The sequences of the oar-miR-200c mimics were as follows: 5'-UAAUAC UGCCGGGUAAUGAUGG-3' (sense), 5'-AUCAUUACCC GGCAGUAUUAU U-3' (antisense); oar-miR-200c inhibitors: CCAUCAUUACCCGGCAGUAUUA; and negative control (NC): 5'-UUCUCCGAACGUG UCACGUTT-3' (sense), 5'-ACGUGACACG UUCGGAGAATT-3' (antisense).

\section{In vitro differentiation}

Embryoid body (EB) formation: 50,000 siPSC/ml was cultured using the pendant drops method for $2 \mathrm{~d}$, then transferred to a bacterial culture dish for further suspension culture for $7 \mathrm{~d}$ (4). Specific gene transcription markers in the endoderm (Foxa2), mesoderm (Actc1), and ectoderm (Neurod) were analyzed by PCR (4). EBs were transferred into a 24-well cell culture plate coated with $0.1 \%$ gelatin and further cultured for $7 \mathrm{~d}$, and the specific gene markers of differentiation of endoderm (SOX17), mesoderm (SMA), and ectoderm (TUJ-1) were analyzed by immunofluorescence.

\section{Bisulfite genomic sequencing}

Total DNA was extracted using the genomic DNA extraction kit (Tiangen, China) and then methylated using an EZ DNA Methylation Gold Kit (Zymo Research, USA). After PCR amplification, DNA fragments were linked to pMD18-T (TaKaRa, China), and the methylation level of $\mathrm{CpG}$ islands in the Nanog promoter region was analyzed by sequencing as previously described (4).

\section{Karyotyping}

Briefly, the siPSCs after 10 generations were treated with $0.2 \mu \mathrm{g} / \mathrm{ml}$ colchicine (Sigma, USA) for $2 \mathrm{~h}$. After TryPLE digestion, $0.075 \mathrm{M} \mathrm{KCl}$ solution was added and kept at $37^{\circ} \mathrm{C}$ for $20 \mathrm{~min}$, then the siPSCs were collected by centrifugation and fixed in methanol : glacial acetic acid $(3: 1)$. Fixation was repeated, then the cells were placed in an ice bath for $20 \mathrm{~min}$. The cells were collected by centrifugation, dropped onto the precooled slides, and then stained with Giemsa for 15 min. Chromosome karyotype images were observed under a microscope.

\section{ZEB1 3'UTR luciferase reporter assay}

The 3'UTR of ZEB1 was PCR-amplified from cDNA derived from SKC. PCR primers used to amplify the Zebl 3'UTR included 5'-CGAGCTCTTGATTAGATGAGCACC TGAC-3' (forward) and 5'-CCCTCGAG TGTTCTACAGT CCAAGGCAAG-3' (reverse), with restriction sites SacI and XhoI on both ends. The PCR conditions were as follows: $94^{\circ} \mathrm{C}$ for $5 \mathrm{~min}$; followed by 35 cycles of $94^{\circ} \mathrm{C}$ for $40 \mathrm{~s}, 55^{\circ} \mathrm{C}$ for $30 \mathrm{~s}$, and $72^{\circ} \mathrm{C} 1 \mathrm{~min}$; and a final extension at $72^{\circ} \mathrm{C}$ for $10 \mathrm{~min}$. Six binding sites between oar-miR-200c and ZEB1 3'UTR were mutated to contain SacI and XhoI restriction sites at both ends, which was synthesized (Sangon Biotech, China). The amplified 3'UTR and 3'UTR mutation were cloned into the double-luciferase reporter gene vector pmirGLO (Promega, USA). HEK293FT cells were seeded into 96-well plates. And when the cells reached $70 \%$ confluency, 100 ng pmiRGLO-ZEB1-3'UTR/ or mutation plasmid and $0.3 \mu 1 \mathrm{miR}-200 \mathrm{c}$ mimics/or inhibitor/or NC (20 $\mathrm{nM})$ were co-transfected using Lipofectamine 2000. The experiment was divided into six groups and performed in triplicate in each group. At $48 \mathrm{~h}$ after transfection, the cells were treated according to the Dual-Luciferase $^{\mathbb{R}}$ Reporter Assay Kit (Promega, USA), and luciferase activity was measured using a BioTek instrument. Relative fluorescence activity was calculated as follows: Relative fluorescence activity $=($ Firefly lucifer-

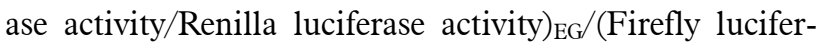
ase activity/Renilla luciferase activity)CG.

\section{Western blotting}

SKCs were transfected with oar-miR-200c mimics (or inhibitor) and collected $48 \mathrm{~h}$ later. After treating with protein lysate (RIPA : PMSF $=100: 1$ ), the supernatant was collected by centrifugation at $12,000 \mathrm{~g}$ for $5 \mathrm{~min}$ at $4^{\circ} \mathrm{C}$. Total protein content was determined by a BCA protein assay kit (Beyotime, China). Approximately $30 \mu \mathrm{g}$ of extracted protein with loading buffer was loaded into wells of a $12 \%$ sodium dodecyl sulfate-polyacrylamide gel (SDS-PAGE) for electrophoresis and then transferred to a nitrocellulose (NC) membrane (Millipore, USA). After blocking, the membranes were treated with anti-ZEB1 ( $1: 3,000$, Abcam), anti- $\beta$-actin $(1: 5,000$, Abcam), and anti-E-cadherin $(1: 2,500$, BD Biosciences) antibodies and incubated overnight at $4^{\circ} \mathrm{C}$. Then, the membranes were treated with horseradish peroxidase-conjugated secondary antibody (Bioworld, China) for $1 \mathrm{~h}$ at $37^{\circ} \mathrm{C}$. The protein bands were visualized using a gel imager (Bio-Rad, USA). 


\section{Statistical analysis}

Statistical analysis was performed using GraphPad Prism8. Paired sample t-tests were used to compare differences between and among groups. Values of $* \mathrm{p}<0.05$ and ${ }^{* *} \mathrm{p}<0.01$ were considered significant and highly significant, respectively.

\section{Results}

\section{Overexpression of miR-200c-141 improves efficiency of siPSC generation}

SKCs were infected with 8 defined factors and miR-200c-141 lentivirus at an MOI $=10$ in the presence of polybrene, then were digested by TryPLE at $2 \mathrm{~d}$ and 10 $\mathrm{d}$ post-infection and passaged in a feed-coated six-well
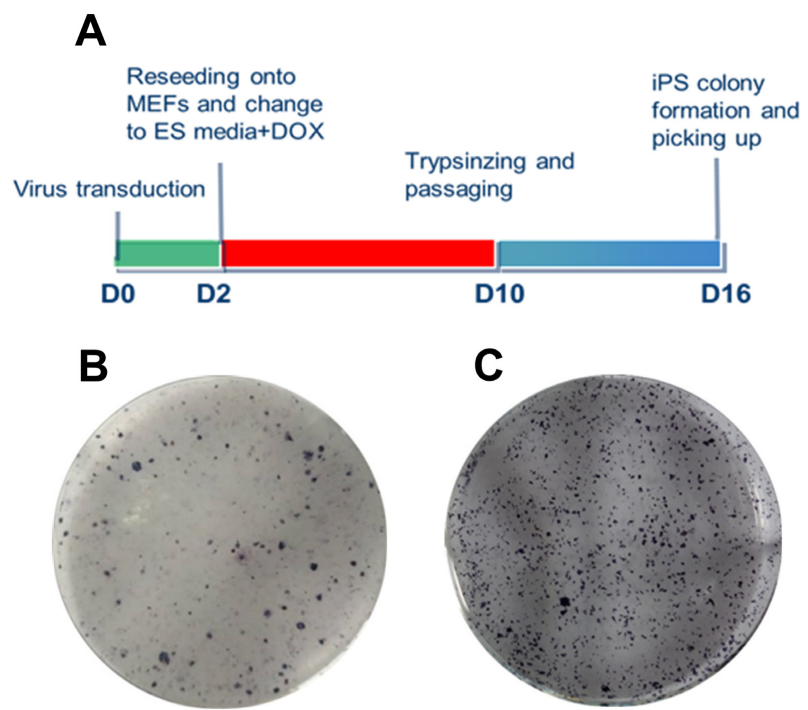

D
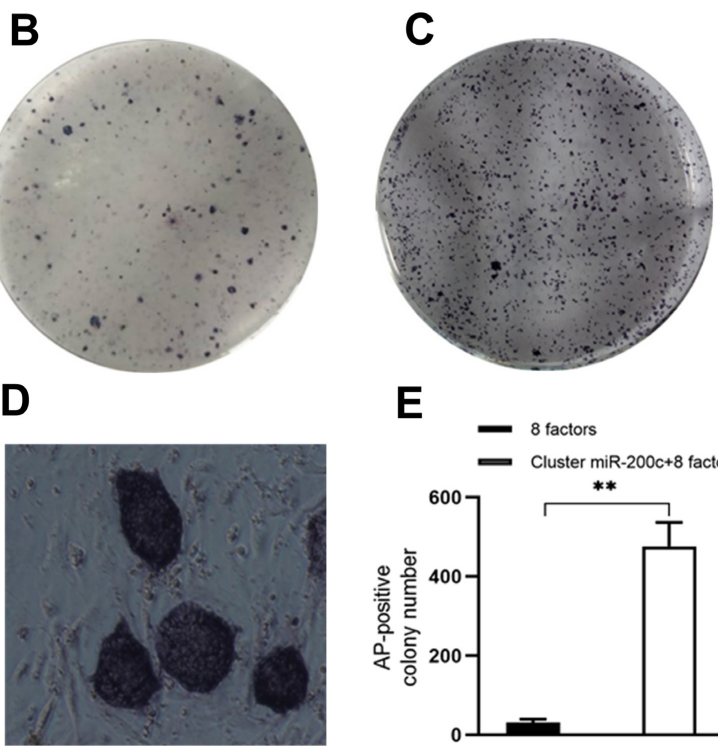

E

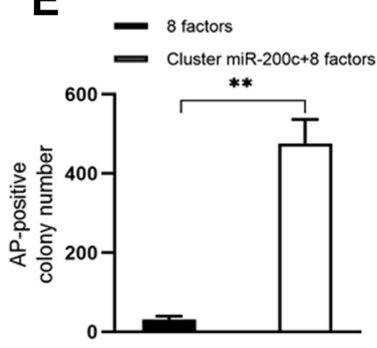

Fig. 1. Process of sheep induced pluripotent stem cell (siPSC) generation and reprogramming efficiency calculation of siPSC. (A) Schematic diagram of sheep kidney cells (SKCs) reprogramming protocol used. (B) AP staining of siPSC generation with 8 factors (Oct4/Sox2/Klf4/c-Myc/Nanog/Lin28/ hTERT/SV40LT). (C) AP staining of siPSC generation with 8 factors plus miR200c-141. (D) Magnification image of representative AP-positive siPSC colonies $(100 \times)$. (E) The number of AP-positive colonies was counted to calculate the reprogramming efficiency. Data presented as the mean \pm standard deviation (SD), $\mathrm{n}=3,{ }^{* *} \mathrm{p}<0.01$. plate for further culture using siPSC medium (Fig. 1A). The experiment showed that the lentivirus system driven by Tet-on carrying Oct4, Sox2, c-Myc, Klf4, Nanog, Lin28, hTERT and SV40LT could reprogram sheep somatic cells into pluripotent cells (Fig. 1B and 1E). Lentivirus-infected cells expressed high levels of green fluorescent protein (GFP) at $48 \mathrm{~h}$ post-infection in group B (Fig. 2A). Numerous circular protrusion colonies with large nucleocytoplasmic ratio and clear boundaries were observed at $16 \mathrm{~d}$ post-infection (Fig. 2B and 2C). Typical colonies were picked and placed into 96-well plates for 5 min with TrypLE. Then, the cells were transferred to a new 96-well plate coated with MEF feeder for further expansion at D16.

AP staining was performed on the remaining colonies in 6-well plate to count the number and calculate the reprogramming efficiency of sheep somatic cells. AP-positive siPSC colonies were dark purple (Fig. $1 \mathrm{~B} \sim \mathrm{D}$ ). Approximately $476 \mathrm{AP}$-positive colonies were derived from 50,000 SKCs infected with a cocktail of lentiviruses (8 defined factors and miR-200c-141 lentivirus), which was 16-fold more than the 8 defined factors in the lentiviral treatment group (Fig. 1B, 1C, and 1E). In addition, the reprogramming efficiency of miR-200c-141 with defined factors was about $0.95 \%(476 / 50,000)$, which highly significantly $(p<0.01)$ improved the generation efficiency of
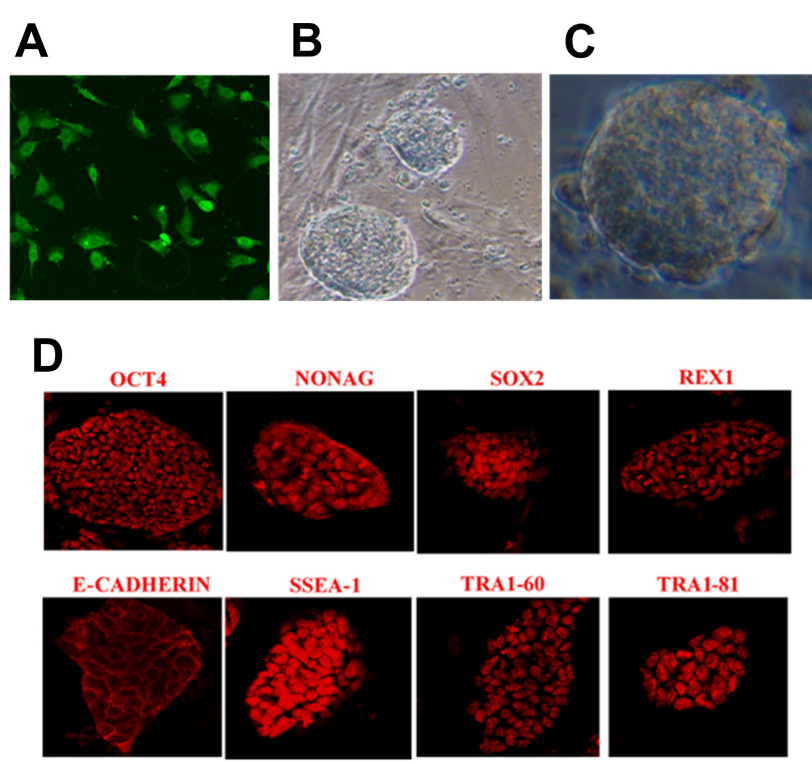

TRA1-81

Fig. 2. Characterization of siPSCs by viral transduction of miR-200c-141 plus 8 factors. (A) SKCs expressed high levels of green fluorescent protein at $48 \mathrm{~h}$ after lentivirus transduction. (B) Typical morphology of siPSC colonies. (C) Magnification of the siPSC colony $(200 \times)$. (D) siPSCs expressed pluripotency markers using immunofluorescence $(200 \times)$. 
siPSCs compared to the single defined factors group (Fig. 1E).

\section{characterization of sheep iPSCs derived from miR-200c-141}

The transcription and expression of pluripotency factors in sheep iPSCs derived from miR-200c-141 and 8 factors

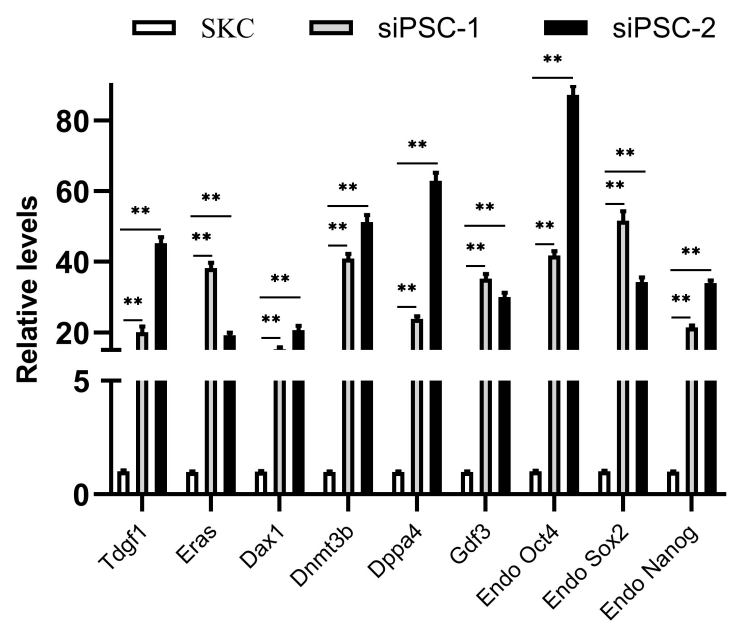

Fig. 3. Relative mRNA expression levels of pluripotency markers in siPSCs by quantitative real-time PCR (qRT-PCR). Data presented as the mean $\pm \mathrm{SD}, \mathrm{n}=3,{ }^{* *} \mathrm{p}<0.01$. were detected. Sheep iPSC colonies were stained with AP, and most of these were purplish black under a microscope after $0.5 \sim 1 \mathrm{~h}$ (Fig. 1D). The experiment results showed that the sheep iPSCs had high-expressed AP, which preliminarily verified the ESC-like characterization of siPSCs. Immunofluorescence revealed that sheep iPSCs expressed pluripotent protein markers similar to ESCs, including OCT4, NANOG, SOX2, REX1, E-cadherin, SSEA-1, TRA1-60, and TRA1-81 (Fig. 2D). Two siPSCs were selected, and the mRNA relative expression levels of endogenous specific pluripotent factors were detected by qRT-PCR. The results showed that the ESC-like endogenous marker genes, including Tdgfl, Eras, Daxl, Dnmt3b, Dppa4, Gdf3, Oct4, Sox2, and Nanog, were all activated in siPSCs, and the mRNA relative expression of these genes was highly significant compared with the primary SKCs $(\mathrm{p}<0.01)$ (Fig. 3).

To examine whether siPSCs could differentiate into cell types of the three germ layers in vitro, the siPSCs were cultured with the pendant drops method for $2 \mathrm{~d}$, then transferred to bacterial culture dish for further suspension culture. After 7 8 days, spherical suspended EB was formed, which was bright and compact, and some EB showed aggregation and adhesion (Fig. 4A). Total RNA of the EBs was extracted and reverse-transcribed into
A

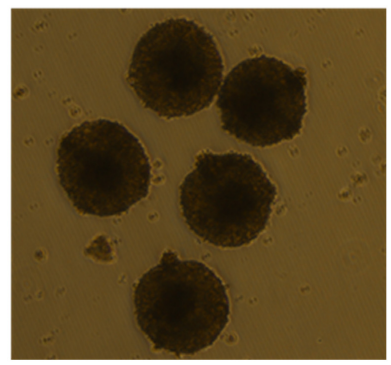

C

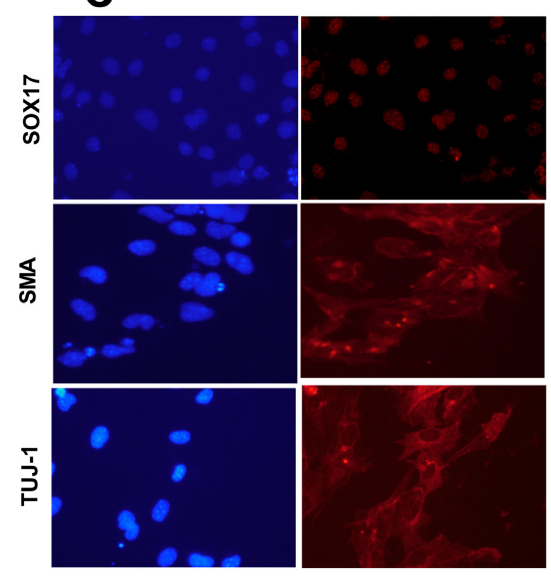

B

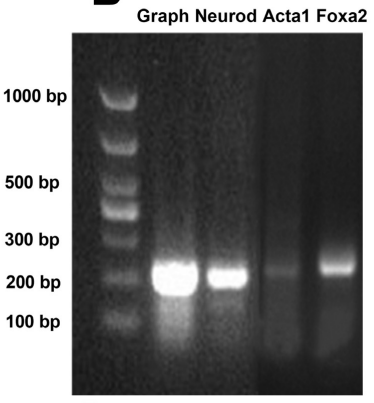

D

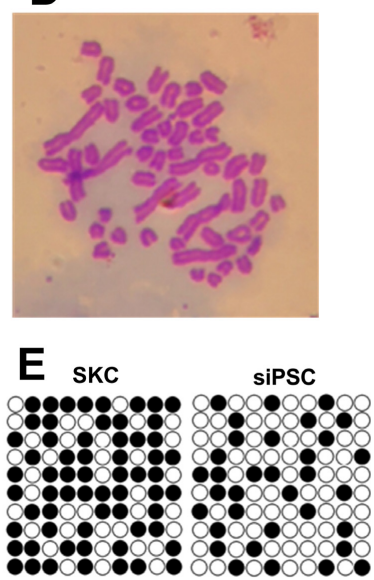

Fig. 4. Characterization and differentiation ability of siPSCs. (A) Representative images of embryoid body (EB) $(100 \times)$. (B) Detection of differentiation markers for the three germ layers in the EB using reverse transcriptase-PCR. (C) Detection of differentiation markers for the three germ layers in the EB using immunofluorescence. (D) Karyotype analysis of siPSC. (E) Bisulfite genomic sequencing of the promoter regions of Nanog (open circles: non-methylation; closed circles: methylation). 
cDNA. Expression of specific genes in the ectodermal (Neurod, 220 bp), mesodermal (Actc1, 237 bp), and endodermal (Foxa2, 254 bp) was detected by PCR. The results showed that all three germ layer markers were transcribed in the EB derived from miR-200c-141 siPSCs (Fig. 4B). Next, the ability of siPSCs to differentiate into three germ layers was assessed at the protein level by immunofluorescence. The results showed that the specific protein markers that were expressed in the ectoderm (TUJ-1), mesoderm (SMA), and endoderm (SOX17) were expressed after EB differentiation (Fig. 4C). These results indicated that siPSCs had the ability to differentiate into three germ layers in vitro.

The methylation of $\mathrm{CpG}$ islands in the Nanog-specific promoter region (5,000-bp before the initial expression of ATG) in sheep iPSCs was assessed by bisulfite genomic sequencing (BSP) (4). The results showed that many sites of $\mathrm{CpG}$ in the Nanog promoter of siPSC were demethylated compared with the initial SKCs (Fig. 4E), indicating that the Nanog gene was in an open and active state and indirectly indicating that SKC was effectively reprogrammed. In addition, siPSCs with more than 10 generations were selected for karyotype analysis. The results showed that the karyotypes of 34 siPSCs were analyzed, and 28 were normal with 54 chromosomes, indicating that the cell karyotypes could be maintained continuously during in- duction (Fig. 4D).

\section{Oar-miR-200c targets the 3'UTR of ZEB1}

Cluster miR-200c-141 contains two miRNAs, namely, miR-200c and miR-141. The seed sequences of miR-200c and miR-141 in sheep were AAUACUG and AACACUG, respectively, with only one base difference, and its target sites may be the same. Therefore, this study focused on the target genes of oar-miR-200c to elucidate the mechanism of miR-200c-141 promoting SKC reprogramming efficiency. The target genes of oar-miR-200c were predicted by the miRbase (www.mirbase.org) and Target Scan (www.targetscan.org) bioinformatics platforms. According to the sequence matching and score of miR-200c and target genes, as well as the signal pathway analysis of target genes, the ZEB1 gene was finally determined and verified.

Total RNA of the SKCs was extracted and reverse-transcribed into cDNA. There are six binding sites between miR-200c and ZEB1 3'UTR. The interaction sequence between miR-200c and ZEB1 3'UTR was amplified by PCR. Mutation interaction sites were designed to form ZEB1 3'UTR mutation sequence (Fig. 5A). ZEB1-3'UTR and ZEB1-3'UTR mutation were linked to dual-luciferase reporter plasmids pmiRGLO, and the sequences were correct as determined by enzyme digestion and sequencing (data not shown). Relative luciferase activity significantly
A

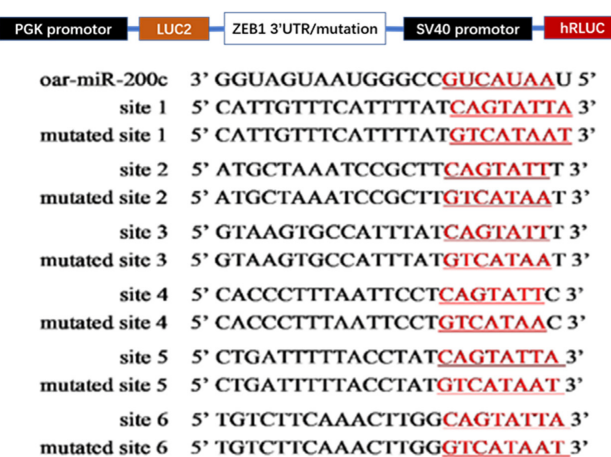

C

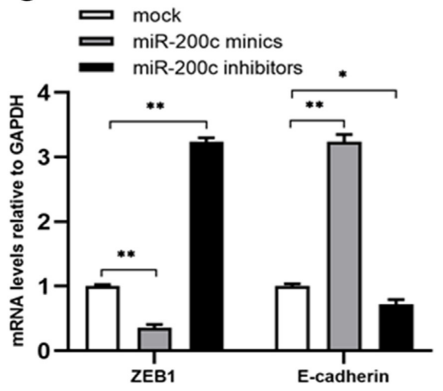

B

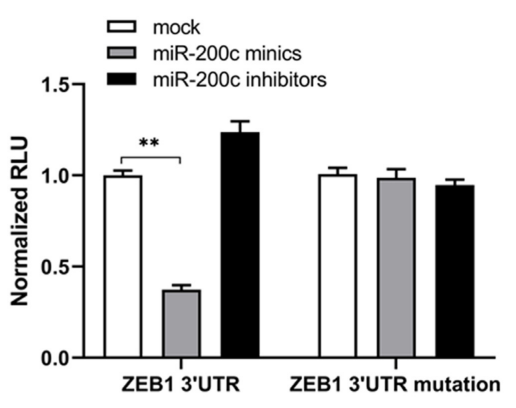

Fig. 5. Oar-miR-200c hinders ZEB1 expression and upregulates E-cadherin expression during SKCs reprogramming. (A) Schematic representation of ZEB1 3'UTR cloned into luciferase reporter vector-pmiRGLO. The 6 binding sites sequence in the $3^{\prime} U T R$ and the corresponding mutations for miR-200c are underlined. (B) miR-200c directly targeted the ZEB1 3'UTR by dual-luciferase reporter assay. (C) Overexpressing miR-200c in SKCs down-regulated of ZEB1 and up-regulated of E-cadherin transcription levels by qRT-PCR. (D) Overexpressing miR-200c in SKCs down-regulated of ZEB1 and up-regulated of E-cadherin protein expression by western blotting. $\beta$-actin is the loading control. Data presented as the mean $\pm \mathrm{SD}, \mathrm{n}=3,{ }^{*} \mathrm{p}<0.05, * * \mathrm{p}<$ 0.01. ZEB1: zinc finger E-box-binding homeobox 1 . 
decreased after co-transfection of oar-miR-200c mimics and pmiRGLO-ZEB1-3'UTR plasmids $(\mathrm{p}<0.01)$, while it was not significantly changed after co-transfection of oar-miR-200c mimics and pmiRGLO-ZEB1-3'UTR-mutation plasmids (Fig. 5B), indicating that oar-miR-200c mimics directly targeted the 3'UTR of MET-related gene ZEB1.

\section{Overexpression of oar-miR-200c inhibits the expression of ZEBI while inducing that of E-cadherin}

To further verify whether miR-200c targeted ZEB1 gene, we transfected oar-miR-200c mimics, oar-miR-200c inhibitor, and NC into SKCs. Then the expression level of ZEB1 was detected by qRT-PCR and western blotting. qRT-PCR results showed that oar-miR-200c mimics significantly decreased the mRNA relative level of ZEB1 $(p<$ 0.01), whereas oar-miR-200c inhibitor transfection significantly increased the mRNA relative level of ZEB1 $(\mathrm{p}<$ 0.01) (Fig. 5C). Western blotting showed that oar-miR-200c mimics-transfected SKCs significantly reduced the protein expression of ZEB1, whereas oar-miR-200c inhibitor transfection significantly increased the protein expression of ZEB1 (Fig. 5D). These results confirmed that oar-miR-200c could down-regulate the expression of ZEB1 gene.

Epithelial-mesenchymal transformation (EMT) requires the upregulation of Smad-dependent critical transcription factors (including ZEB1, Snail, and Twist) in the TGF- $\beta$ signaling pathway, which typically activates EMT by binding to the promoter of E-cadherin to inhibit its expression. However, overexpression of E-cadherin can promote mesenchymal-epithelial transformation (MET). Moreover, MET is an important event and marker of cell reprogramming at the initial stage. Therefore, we inferred that the decrease of ZEB1 could increase the expression of E-cadherin, and thus promoted the sheep somatic reprogramming process. Results showed that mRNA relative level $(\mathrm{p}<$ 0.01 ) and protein expression of E-cadherin were significantly increased after oar-miR-200c mimic transfection, whereas oar-miR-200c inhibitor significantly decreased the expression of E-cadherin (Fig. 5C and 5D).

\section{Discussion}

iPSCs have been successfully utilized in mouse and human, bringing the research into a new era in the field of stem cells. However, the study of sheep iPSCs is still in its infancy, and its induction efficiency is low $(4,5,8)$, and thus it is difficult to obtain fully reprogrammed iPSCs, hindering systematic and in-depth understanding of the reprogramming process and mechanism of sheep cells. Therefore, screening miRNAs that can promote sheep somatic cell reprogramming and exploring its reprogramming mechanism is of great significance in elucidating the reprogramming mechanism of large livestock cells and the establishment of sheep iPSCs or ESC lines. It will have broad application prospects for high-quality sheep breeding, as well as in the biomedical and pharmaceutical industries.

Studies have shown that iPSCs can be generated by different tissue cells, but with different efficiency. In our previous study, we compared the reprogramming ability of skin fibroblasts, sheep kidney cells (SKCs) and bone marrow stromal cells (BMSCs) using the same defined factors, and found that SKC had a stronger ability to reprogram into pluripotency (6). Therefore, SKCs were selected as the starting cell for reprogramming in this study. At present, the iPSC technology is extensively utilized, and induction efficiency and safety of iPSC are constantly improving. Small molecule chemicals, mRNAs and miRNAs do not need to integrate exogenous transcription factors into the genome, which is an ideal means to establish iPSC cell lines. The use of miRNAs to improve the induction efficiency has been studied in mouse and human, but less so in large animals. Expression of cluster miR-302/367 using lentiviral vectors in mouse and human somatic cells is 100 -fold more efficient than using four transcription factors to generate iPSCs (15). miR-106a-363 and miR-302-367 also promote the efficiency of mouse iPSCs using three factors (20). The cluster miR-302/367 (21), miR-302a/b, and miR-200 family (22) could significantly promote the generation of pig iPSC colonies, but had no effect for bovine cell reprogramming (23). The expression of cluster miR-302/367 and bovine OSKM using lentiviral vectors induces primed or naive goat iPSCs with positive $\mathrm{AP}$ staining $(24,25)$. In this study, cluster miR-200c-141 combined with 8-factor lentivirus infection of SKCs increased the reprogramming efficiency to $0.95 \%$. Compared with the eight-factor induction alone, miR-200c-141 increased the efficiency by about 16-fold. The obtained siPSCs had mESC-like round hump colonies, but there were also a large number of primed-like flat colonies. The effect of cluster miR-302/367 on siPSC generation was also measured in our laboratory, and no significant promoting effect was observed (results not shown).

miR-200c has an important relationship with TGF- $\beta$ signaling pathway and MET process and has been studied extensively in the field of oncology. The TGF- $\beta$ signaling pathway can activate Smad-dependent and non-Smad-dependent signals through type I and II receptor complexes, 
thereby regulating cell proliferation and differentiation (26). Smad-dependent signaling pathways are involved in the EMT process, which require the upregulation of important transcription factors during, including ZEB1, ZEB2, Snail, Slug, and Twist (27, 28). These transcription factors activated EMT by binding to the promoter regions of many epithelial-specific genes including the E-cadherin, to inhibit the synthesis of adhesion proteins between cells (29). Studies have found that activation of the TGF$\beta$-Smad3/4 signaling pathway can inhibit the expression of miR-200c (30). TGF- $\beta$ and TNF- $\alpha$ can induce the formation of EMT in colorectal cancer cells, pancreatic cancer cells, and breast cancer cells by upregulating ZEB1, while overexpression of miR-200c or silencing of ZEB1 can partially inhibit the EMT process and reduce the metastatic ability of tumor cells (31). Research on EMT found that both miR-200c and ZEB-1/2 constitute a double-negative feedback loop $(32,33)$.

Somatic cells are reprogrammed into pluripotent cells, and MET is considered an important checkpoint in the reprogramming process (34). EMT regulated by TGF- $\beta$ signaling is an obstacle to somatic cell reprogramming in mouse and human, and its inhibitors enhance reprogramming $(35,36)$. In the initial stage of mouse cell reprogramming, the MET process can be driven by the BMP-Smad signal to improve the efficiency of reprogramming after overexpressing mmu-miR-200 and mmu-miR-205 (37). $\mathrm{ZEB} 1$ and $\mathrm{ZEB} 2$ are the key transcriptional repressors of E-cadherin (38). Overexpression of hsa-miR-205 and hsa-miR-200 family in canine mesenchymal cells initiate MET by directly targeting the inhibition of ZEB1 and ZEB2, resulting in E-cadherin activation (39). In addition, overexpression of mmu-miR-200 in mouse epithelial cells could directly target and inhibit ZEB1 and ZEB2, and then prevent EMT by inhibiting the TGF- $\beta$ pathway (40). This study also confirmed that miR-200c can activate E-cadherin by inhibiting the expression of ZEB1, thereby initiating the MET process in the sheep somatic cell reprogramming. miR-200c plays an important role in the siPSC generation. The miR-200c-ZEB1-MET signal is present not only in mouse but also in the reprogramming process of sheep somatic cells.

In conclusion, the lentivirus system carrying Oct4, Sox2, c-Myc, Klf4, Nanog, Lin28, hTERT and SV40LT could reprogram sheep somatic cells into pluripotent cells. Overexpression of miR-200c-141 in combination with OSKMNLST improves reprogramming efficiency. OarmiR-200c targets the ZEB1 3'UTR sequences and decreases its expression, but could increase the expression of E-cadherin. Oar-miR-200c enhances the MET process by affecting the TGF- $\beta$ signaling pathway, thus improving the efficiency of sheep somatic cell reprogramming.

\section{Acknowledgments}

We thank Prof. Lei Xiao for providing the lentivirus system. We thank addgene for providing the lentiviral vectors. We thank Associate Prof. Huijun Shi for her guidance on the experimental techniques. This research was supported by open project of State key Laboratory of Sheep Genetic Improvement and Healthy Production (MYSKLKF201901); The Bingtuan Science and Technology Cooperation Program (2018BC011).

\section{Potential Conflict of Interest}

The authors have no conflicting financial interest.

\section{References}

1. Takahashi K, Yamanaka S. Induction of pluripotent stem cells from mouse embryonic and adult fibroblast cultures by defined factors. Cell 2006;126:663-676

2. Yu J, Vodyanik MA, Smuga-Otto K, Antosiewicz-Bourget J, Frane JL, Tian S, Nie J, Jonsdottir GA, Ruotti V, Stewart $\mathrm{R}$, Slukvin II, Thomson JA. Induced pluripotent stem cell lines derived from human somatic cells. Science 2007;318:1917-1920

3. Li Y, Cang M, Lee AS, Zhang K, Liu D. Reprogramming of sheep fibroblasts into pluripotency under a drug-inducible expression of mouse-derived defined factors. PLoS One 2011;6:e15947

4. Bao L, He L, Chen J, Wu Z, Liao J, Rao L, Ren J, Li H, Zhu H, Qian L, Gu Y, Dai H, Xu X, Zhou J, Wang W, Cui C, Xiao L. Reprogramming of ovine adult fibroblasts to pluripotency via drug-inducible expression of defined factors. Cell Res 2011;21:600-608

5. Liu J, Balehosur D, Murray B, Kelly JM, Sumer H, Verma PJ. Generation and characterization of reprogrammed sheep induced pluripotent stem cells. Theriogenology 2012; 77:338-346.e1

6. Shi H, Fu Q, Li G, Ren Y, Hu S, Ni W, Guo F, Shi M, Meng L, Zhang H, Qiao J, Guo Z, Chen C. Roles of p53 and ASF1A in the reprogramming of sheep kidney cells to pluripotent cells. Cell Reprogram 2015;17:441-452

7. Sartori C, DiDomenico AI, Thomson AJ, Milne E, Lillico SG, Burdon TG, Whitelaw CB. Ovine-induced pluripotent stem cells can contribute to chimeric lambs. Cell Reprogram 2012;14:8-19

8. Su Y, Zhu J, Salman S, Tang Y. Induced pluripotent stem cells from farm animals. J Anim Sci 2020;98:skaa343

9. Pfaff N, Moritz T, Thum T, Cantz T. miRNAs involved in the generation, maintenance, and differentiation of pluripotent cells. J Mol Med (Berl) 2012;90:747-752

10. Choi E, Choi E, Hwang KC. MicroRNAs as novel regulators of stem cell fate. World J Stem Cells 2013;5:172-187 
11. Houbaviy HB, Murray MF, Sharp PA. Embryonic stem cell-specific MicroRNAs. Dev Cell 2003;5:351-358

12. Suh MR, Lee Y, Kim JY, Kim SK, Moon SH, Lee JY, Cha KY, Chung HM, Yoon HS, Moon SY, Kim VN, Kim KS. Human embryonic stem cells express a unique set of microRNAs. Dev Biol 2004;270:488-498

13. Judson RL, Babiarz JE, Venere M, Blelloch R. Embryonic stem cell-specific microRNAs promote induced pluripotency. Nat Biotechnol 2009;27:459-461

14. Li N, Long B, Han W, Yuan S, Wang K. microRNAs: important regulators of stem cells. Stem Cell Res Ther 2017;8:110

15. Anokye-Danso F, Trivedi CM, Juhr D, Gupta M, Cui Z, Tian Y, Zhang Y, Yang W, Gruber PJ, Epstein JA, Morrisey EE. Highly efficient miRNA-mediated reprogramming of mouse and human somatic cells to pluripotency. Cell Stem Cell 2011;8:376-388

16. Wang G, Guo X, Hong W, Liu Q, Wei T, Lu C, Gao L, Ye D, Zhou Y, Chen J, Wang J, Wu M, Liu H, Kang J. Critical regulation of miR-200/ZEB2 pathway in Oct4/Sox2-induced mesenchymal-to-epithelial transition and induced pluripotent stem cell generation. Proc Natl Acad Sci U S A 2013;110:2858-2863

17. Miyoshi N, Ishii H, Nagano H, Haraguchi N, Dewi DL, Kano Y, Nishikawa S, Tanemura M, Mimori K, Tanaka F, Saito T, Nishimura J, Takemasa I, Mizushima T, Ikeda M, Yamamoto H, Sekimoto M, Doki Y, Mori M. Reprogramming of mouse and human cells to pluripotency using mature microRNAs. Cell Stem Cell 2011;8:633-638

18. Mallanna SK, Rizzino A. Emerging roles of microRNAs in the control of embryonic stem cells and the generation of induced pluripotent stem cells. Dev Biol 2010;344:16-25

19. Livak KJ, Schmittgen TD. Analysis of relative gene expression data using real-time quantitative PCR and the 2(-Delta Delta C(T)) method. Methods 2001;25:402-408

20. Liao B, Bao X, Liu L, Feng S, Zovoilis A, Liu W, Xue Y, Cai J, Guo X, Qin B, Zhang R, Wu J, Lai L, Teng M, Niu L, Zhang B, Esteban MA, Pei D. MicroRNA cluster 302-367 enhances somatic cell reprogramming by accelerating a mesenchymal-to-epithelial transition. J Biol Chem 2011;286:17359-17364

21. Du X, Feng T, Yu D, Wu Y, Zou H, Ma S, Feng C, Huang Y, Ouyang H, Hu X, Pan D, Li N, Wu S. Barriers for deriving transgene-free pig iPS cells with episomal vectors. Stem Cells 2015;33:3228-3238

22. Ma K, Song G, An X, Fan A, Tan W, Tang B, Zhang X, Li Z. miRNAs promote generation of porcine-induced pluripotent stem cells. Mol Cell Biochem 2014;389:209-218

23. Pillai VV, Kei TG, Reddy SE, Das M, Abratte C, Cheong $\mathrm{SH}$, Selvaraj V. Induced pluripotent stem cell generation from bovine somatic cells indicates unmet needs for pluripotency sustenance. Anim Sci J 2019;90:1149-1160

24. Tai D, Liu P, Gao J, Jin M, Xu T, Zuo Y, Liang H, Liu D. Generation of Arbas cashmere goat induced pluripotent stem cells through fibroblast reprogramming. Cell Reprogram 2015;17:297-305
25. Sandmaier SE, Nandal A, Powell A, Garrett W, Blomberg L, Donovan DM, Talbot N, Telugu BP. Generation of induced pluripotent stem cells from domestic goats. Mol Reprod Dev 2015;82:709-721

26. Bobik A. Transforming growth factor-betas and vascular disorders. Arterioscler Thromb Vasc Biol 2006;26:1712-1720

27. Moreno-Bueno G, Cubillo E, Sarrió D, Peinado H, Rodríguez-Pinilla SM, Villa S, Bolós V, Jordá M, Fabra A, Portillo F, Palacios J, Cano A. Genetic profiling of epithelial cells expressing E-cadherin repressors reveals a distinct role for Snail, Slug, and E47 factors in epithelial-mesenchymal transition. Cancer Res 2006;66:9543-9556

28. Shirakihara T, Saitoh M, Miyazono K. Differential regulation of epithelial and mesenchymal markers by deltaEF1 + proteins in epithelial mesenchymal transition induced by TGF-beta. Mol Biol Cell 2007;18:3533-3544

29. Peinado H, Olmeda D, Cano A. Snail, Zeb and bHLH factors in tumour progression: an alliance against the epithelial phenotype? Nat Rev Cancer 2007;7:415-428

30. Bryant JL, Britson J, Balko JM, Willian M, Timmons R, Frolov A, Black EP. A microRNA gene expression signature predicts response to erlotinib in epithelial cancer cell lines and targets EMT. Br J Cancer 2012;106:148-156

31. Burk U, Schubert J, Wellner U, Schmalhofer O, Vincan E, Spaderna S, Brabletz T. A reciprocal repression between ZEB1 and members of the miR-200 family promotes EMT and invasion in cancer cells. EMBO Rep 2008;9:582-589

32. Bracken CP, Gregory PA, Kolesnikoff N, Bert AG, Wang J, Shannon MF, Goodall GJ. A double-negative feedback loop between ZEB1-SIP1 and the microRNA-200 family regulates epithelial-mesenchymal transition. Cancer Res 2008;68:7846-7854

33. Brabletz S, Brabletz T. The ZEB/miR-200 feedback loop--a motor of cellular plasticity in development and cancer? EMBO Rep 2010;11:670-677

34. Lamouille S, Xu J, Derynck R. Molecular mechanisms of epithelial-mesenchymal transition. Nat Rev Mol Cell Biol 2014;15:178-196

35. Ichida JK, Blanchard J, Lam K, Son EY, Chung JE, Egli D, Loh KM, Carter AC, Di Giorgio FP, Koszka K, Huangfu D, Akutsu H, Liu DR, Rubin LL, Eggan K. A small-molecule inhibitor of tgf-Beta signaling replaces sox2 in reprogramming by inducing nanog. Cell Stem Cell 2009;5:491-503

36. Lin T, Ambasudhan R, Yuan X, Li W, Hilcove S, Abujarour R, Lin X, Hahm HS, Hao E, Hayek A, Ding S. A chemical platform for improved induction of human iPSCs. Nat Methods 2009;6:805-808

37. Samavarchi-Tehrani P, Golipour A, David L, Sung HK, Beyer TA, Datti A, Woltjen K, Nagy A, Wrana JL. Functional genomics reveals a BMP-driven mesenchymalto-epithelial transition in the initiation of somatic cell reprogramming. Cell Stem Cell 2010;7:64-77

38. Peter ME. Let-7 and miR-200 microRNAs: guardians against pluripotency and cancer progression. Cell Cycle 2009;8:843-852 
39. Gregory PA, Bert AG, Paterson EL, Barry SC, Tsykin A, Farshid G, Vadas MA, Khew-Goodall Y, Goodall GJ. The miR-200 family and miR-205 regulate epithelial to mesenchymal transition by targeting ZEB1 and SIP1. Nat Cell Biol 2008;10:593-601
40. Korpal M, Lee ES, Hu G, Kang Y. The miR-200 family inhibits epithelial-mesenchymal transition and cancer cell migration by direct targeting of E-cadherin transcriptional repressors ZEB1 and ZEB2. J Biol Chem 2008;283:1491014914 